\title{
T wie tempora mutantur
}

Dominik Heim

Korrespondenz:

PD Dr. med. Dominik Heim

Chefarzt Chirurgie

Spital Frutigen

CH-3714 Frutigen

dominik.heim[at]spitalfmi.ch
Es gibt bekanntlich das Frustessen. Das ist zu unterscheiden vom schwelgerischen, tödlichen Überessen in «La grande bouffe» von Marco Ferreri - der galt als Skandalfilm 1973, etwa so, wie dies aktuell von «Feuchtgebiete» behauptet wird. Und so muss es dann in Analogie dazu auch ein Frustschreiben geben (dafür eignen sich Mails besonders gut), aber das hier ist kein Frustschreiben, wohl aber ein Schreiben über Frust. Und wenn es dann am Ende des Mails noch heisst «Tempora mutantur, nos et mutamur in illis», dann schwingt da auch die Resignation unverkennbar mit. Eigentlich hatte ja zwar Kaiser Lothar I. recht, die Zeiten haben sich seit dem 9. Jahrhundert wirklich geändert und wir uns mit ihnen, aber da waren sicher auch aktive Veränderungen gemeint, jetzt geht's aber passiv ab in die Resignation.

Die Sachlage: Eine Fortbildung ist geplant. Sie kommt wegen mangelnder Beteiligung kurzfristig nicht zustande: «Offensichtlich besteht ein gewisses Desinteresse an Weiterbildungen (...) von 5 Assistentenstellen haben wir momentan nur 3 besetzt, davon ist eine Kollegin gerade im «Burn-out und ein Assistent ist im Urlaub» ist ein Grund für das Fernbleiben. «Wir haben eine momentane Knappheit an Assistenten nach 2 Abgängen, zum andern gäbe es 2 Kandidaten, welche ihre Freizeit schon anderweitig verplant haben ... sic», ist eine andere Erklärung. «Der letzte Assistent hat nach 2 Wochen gekündigt - wir haben hier Zustände wie im Krieg - ich kann und mag nicht mehr und habe ebenfalls gekündigt.» Und dann eben noch das: «Die Assistenten sind heute, wenn nicht in der Arbeitsschicht eingeteilt, am Kompensieren oder sonst am Überzeit abbauen. Wahrscheinlich bleibt uns nichts anderes übrig, als uns der Zeit anzupassen.» Im Klartext und korrekt übersetzt: «Nos et mutamur in illis!»

Bitter klingt das, bitter ist das, denn «irgendwie frage ich mich, wer dann irgendeinmal, wenn ich mit dem Motorrad stürzen sollte, mich operieren wird», endet ein Mail. Werden wir da also mit der gesetzlichen Arbeitszeitbeschränkung, mit den neuen Arbeitsmodellen, die Familie/Freizeit («family matters» schreibt das Forum Junger Chirurgen der SGC) und Beruf endlich unter einen Hut bringen sollen, in den Strudel der medizinischen Unterversorgung gezogen? Und wir akzeptieren das einfach? Weil der Zeitgeist das so will?

Weil «The times they are achangin'», wie Bob Dylan 1964 (scheinbar in weiser Voraussicht) gesungen hat? Rauher, marginaler ist seine Stimme heute geworden, seine Sätze noch mehr gesprochen, gekrächzt. Und mit «Tempest» hat er dann 2012 (schon) wieder einen Sturm, diesmal der Bewunderung, provoziert. 1970 war es noch ein Sturm der Entrüstung. «What's this shit», schrieb MusikkritikerGuru Greil Marcus damals beim Erscheinen von «Self portrait», und der Satz wurde Kult. Denn man erwartete einen zornigen Dylan mit Protestsongs in dieser Zeit des Vietnamkrieges, und es kam eine Sammlung von Coverversionen von traditionellen Folk- und Countrystücken, Liveaufnahmen (vom ebenfalls sehr umstrittenen Auftritt auf der Isle of Wight) und wenigen Eigenkompositionen. Dylan habe es direkt darauf angelegt, das Publikum vor den Kopf zu stossen, wurde vermutet. Jetzt ist dieses Album mit neuen Versionen der Lieder als "another self portrait» in den Läden. Die Kritiker rätseln wieder, die Dylanologen interpretieren wieder (Bob Dylan selbst sagt meist ja gar nichts - bekanntlich gibt es auch keine Texte auf seinen Alben), und auch Marcus befindet jetzt (retrospektiv), dass diese alten Lieder in den neuen Versionen «unreleased» und «alternate version» nun doch mehr sind als nur enttäuschende Zeitdokumente. Aus «shit» wurde Bewunderung und Respekt.

Am Boden zerstört war Dylan damals 1970 nicht durch diesen Verriss. Nur Monate später kam noch «New morning», doch dann gab es für 4 Jahre keinen öffentlichen Dylan mehr - bis zu «Planet waves». Vielleicht täte dies der Medizin auch gut. Die Arbeitsauffassungen scheinen inkompatibel, die Ressentiments gross, der Frust da. Recht oder unrecht, richtig oder falsch, diese Beurteilung sollte, muss vertagt werden. Das Problem ist einfach, dass man «nachher immer schlauer ist», dass es aber bis zur Retrospektion offensichtlich bis zu 40 Jahre dauern kann.

Nur, hier geht es nicht um ein Album, um ein ein-

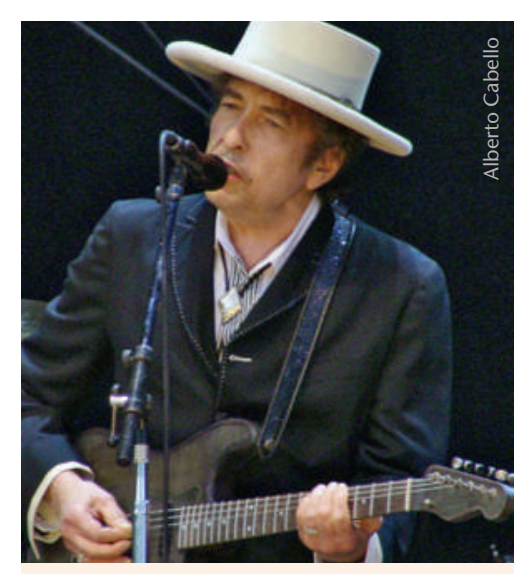

Schon Bob Dylan wusste: «The times they are a-changin'». ziges geniales Schicksal (das sich nicht unterkriegen liess). Hier geht es auch nicht um uns Ärzte, sondern um jene, die sich uns anvertrauen. Resignation und Frust sind sicher nicht die richtige Reaktion, dieses offensichtliche Generationenproblem zu lösen. Aber das sei dann kritisch doch noch (aus meiner Warte) angefügt: Hedonismus pur zerstört! Widerspreche ich da Dylan?

"Come mothers and fathers ... your old road is rapidly agin', Please get out of the new one, if you can't lend your hand, for the times they are changin'.» 